\title{
FK506 suppresses hypoxia-induced inflammation and protects tight junction function via the CaN-NFATc1 signaling pathway in retinal microvascular epithelial cells
}

\author{
YAGUANG HU* ${ }^{*}$ BOYU YANG* ${ }^{*}$ YUE XU, LI JIANG, CHING-KIT TSUI and XIAOLING LIANG \\ State Key Laboratory of Ophthalmology, Zhongshan Ophthalmic Center, \\ Sun Yat-sen University, Guangzhou, Guangdong 510000, P.R. China
}

Received September 15, 2016; Accepted May 24, 2017

DOI: $10.3892 / \mathrm{mmr} .2017 .7475$

\begin{abstract}
The present study aimed to identify whether FK506 suppresses hypoxia-induced inflammation and protects tight junction function via the calcineurin-nuclear factor of activated T-cells 1 (CaN-NFATc1) signaling pathway in mouse retinal microvascular endothelial cells (mRMECs). The mRMECs were treated with FK506 at different concentrations following the induction of hypoxia. Trans-epithelial electrical resistance (TEER) and cell permeability were examined to measure the integrity of the tight junctions. The concentrations of inflammatory cytokines were measured using reverse transcription-quantitative polymerase chain reaction analysis and enzyme-linked immunosorbent assays. The protein expression levels of zonula occludens-1 (ZO-1) and nuclear factor of activated T-cell 1 (NFATc1) were identified using immunofluorescent microscopy and western blot analysis. The TEER value was decreased following hypoxia, but increased following treatment with FK506 (1 and $10 \mu \mathrm{M})$ for 24 and $48 \mathrm{~h}$. The protein expression of ZO-1 was also increased following FK506 treatment for $24 \mathrm{~h}$ at 1 and $10 \mu \mathrm{M}$. By contrast, following treatment with FK506 (1 and $10 \mu \mathrm{M})$ for 24 and $48 \mathrm{~h}$, the elevated cell permeability in the hypoxia group was significantly downregulated. Similarly, the concentrations of inflammatory cytokines, including cyclooxygenase-2, inducible nitric oxide synthase, monocyte chemoattractant protein-1, interleukin-6, intercellular adhesion molecule-1 and vascular cell adhesion molecule-1, were downregulated following treatment with FK506 for $24 \mathrm{~h}$ at 1 and $10 \mu \mathrm{M}$. Following treatment
\end{abstract}

Correspondence to: Dr Xiaoling Liang, State Key Laboratory of Ophthalmology, Zhongshan Ophthalmic Center, Sun Yat-sen University, 54 Xianlie Road, Guangzhou, Guangdong 510000, P.R. China

E-mail: liangxlsums@qq.com

*Contributed equally

Key words: FK506, hypoxia, inflammation, tight junction, calcineurin-nuclear factor of activated T-cells 1 signaling pathway with FK506, the level of total NFATc1 was downregulated and the level of phosphorylated NFATc1 was upregulated. Taken together, FK506 suppressed injury to the tight junctions and downregulated the expression of inflammatory cytokines in hypoxia-induced mRMECs via the CaN-NFATc1 signaling pathway. This suggests a potentially effective therapy for hypoxia-induced retinal microangiopathy.

\section{Introduction}

Diabetic retinopathy (DR) and retinopathy of prematurity (ROP) are types of retinal microangiopathy, which involve blood-retina barrier (BRB) injury and the occurrence of inflammation. In addition to the BRB injury and inflammatory cytokine upregulation, retinal neovascularization (RNV) appears, resulting in bleeding, seepage and hyperplasia, and causing severe vision loss. According to previous reports, RNV-associated diseases are one of the most serious irreversible diseases, which can cause blindness, worldwide $(1,2)$. Anti-vascular endothelial growth factor (VEGF) drugs, including ranibizumab, are the most effective therapeutic drugs at present. However, their clinical application is restricted as patients require multiple injections, which may increase the risk of infections and increases economic burden (3). Therefore, details investigations of the pathogenesis of $\mathrm{RNV}$, and the identification of more effective and economic therapeutic strategies are urgently required to overcome these problems.

Previous studies have shown that chronic hypoxia of the retina is a basic histopathological change in retinal microangiopathies. VEGF, released by the hypoxic retina, is the key trigger factor in neovascularization and inflammation. Calcineurin is distributed on endothelial cells, which can be activated by VEGF. The activation of calcineurin can further initiate the dephosphorylation of NFATc1, which transfers into the nucleus and upregulates the expression of certain inflammatory cytokines, including interleukin (IL)-8, IL-2 and cyclooxygenase-2 (COX-2). As the inflammatory cytokines are upregulated, the vascular walls are altered. The typical histopathological changes include loss of endothelial cells and thickening of the basilar membrane (4), which forms tight junctions. The tight junctions between endothelial cells 
are important in the retinal barrier, which are predominantly destroyed in retinal microangiopathy. The destruction to tight junctions renders the barriers of the retina, particularly the inner BRB, seriously damaged.

FK506, an immunosuppressive drug, is used for as a treatment following transplantation, for atopic dermatitis and rheumatoid arthritis (5-7). FK506 binds to FK506-binding proteins and forms complexes. These complexes then bind to calcineurin, leading to inhibition of the dephosphorylation of NFATc1, which suppresses the expression of inflammatory cytokines and T cell activation (8). FK506 can effectively downregulate the infiltration of inflammatory cells and the expression of E-selectin, intercellular cell adhesion molecule-1 (ICAM-1) and vascular cell adhesion molecule-1 (VCAM-1) in inflamed tissues in vivo $(9,10)$. FK506 has been shown to be closely associated with thrombotic microangiopathy $(11,12)$, however, the direct effects of FK506 on retinal microvascular endothelia cells (RMECs) remain to be fully elucidated. In order to determine an effective treatment for RNV diseases, the present study investigated whether FK506 can suppress hypoxia-induced inflammation and protect tight junction function via the calcineurin-nuclear factor of activated T-cells 1 (CaN-NFATc1) signaling pathway in RMECs.

\section{Materials and methods}

Mouse (m)RMEC culture. The mRMECs (cat. no. CD-1065) were purchased from Cell Biologics, Inc. (Chicago, IL, USA). The mRMECs were cultured in a $100 \mathrm{~mm}$ collagen-coated culture dish containing $10 \mathrm{ml}$ supplemented medium (RPMI-1640; Hyclone; GE Healthcare Life Sciences, Logan, UT, USA) with $20 \%$ fetal bovine serum (16000044; Gibco; Thermo Fisher Scientific, Inc., Waltham, MA, USA) and maintained at $37^{\circ} \mathrm{C}$ in a humidified atmosphere of $5 \% \mathrm{CO}_{2}$ and $95 \%$ air. The culture medium was replaced every 2 days. The cells used in the present study were between passages 3 and 4 . When the cells had grown to $70 \%$ confluence, they were cultured under hypoxic conditions $\left(93 \% \mathrm{~N}_{2}, 5 \% \mathrm{CO}_{2}\right.$, $2 \% \mathrm{O}_{2}$ ) at $37^{\circ} \mathrm{C}$ for $24 \mathrm{~h}$. Then cells were then washed twice with phosphate-buffered saline (PBS) and then exposed to FK506 (F4679; Sigma-Aldrich; Merck KGaA, Darmstadt, Germany) at different concentrations $(0.1,1$ and $10 \mu \mathrm{M})$ for 24 and 48 h, respectively. FK506 was dissolved in dimethyl sulfoxide $(<0.1 \%)$, which caused no deleterious effect on the viability of the mRMECs in preliminary experiments.

Measurement of trans-epithelial electrical resistance (TEER). The permeability of the mRMEC monolayers was assessed via TEER measurement. The TEER across the endothelial cell layer was calculated using a Millicell-ERS Voltohmmeter (EMD Millipore, Bedford, MA, USA). Briefly, the mRMECs were seeded onto a membrane at a density of $3 \times 10^{5}$ cells $/ \mathrm{cm}^{2}$. The TEER measurements were implemented following treatment of the cells with FK506 at different concentrations $(0.1,1$ and $10 \mu \mathrm{M})$. The TEER value of the blank Transwell membrane was used as a blank value, and was subtracted from the sample value. The TEER values were calculated as $\Omega \times \mathrm{cm}^{2}$ and presented as the mean \pm standard error of mean of three independent experiments. Each cell monolayer with a TEER value $>100 \Omega \times \mathrm{cm}^{2}$ was considered to contain tight junctions.

Measurement of cell permeability using fluorescein isothiocyanate (FITC)-dextran. The mRMECs were plated in $12 \mathrm{~mm}$ diameter Transwell polyethylene terephthalate membrane inserts $(0.4 \mu \mathrm{m}$ pore size $)$ at a density of $2 \times 10^{5}$ per insert. The permeability was measured using FITC-dextran, with $1 \mathrm{mg} / \mathrm{ml}$ FITC-dextran added to the apical compartment $4 \mathrm{~h}$ prior to the assessment. The samples were collected $(25 \mu \mathrm{l})$ from the lower compartment for assessment, and PBS was added to $250 \mu \mathrm{l}$. The fluorescence was measured on a fluorescence luminometer at wavelengths of $492 \mathrm{~nm}$ (excitation) and $520 \mathrm{~nm}$ (emission).

Western blot analysis. The cells were lysed on ice for $20 \mathrm{~min}$ using RIPA lysis buffer with protease inhibitor, and were centrifuged at $13,400 \mathrm{x}$ g at $4^{\circ} \mathrm{C}$ for $10 \mathrm{~min}$. The supernatant was collected and total protein concentration was measured by BCA protein assay (Beyotime Institute of Biotechnology, Shanghai, China). The proteins were then boiled in sodium dodecyl sulfate (SDS) sample buffer for $10 \mathrm{~min}$ and then equal amounts of lysate protein (10 $\mu \mathrm{l} /$ lane) were added to $10 \%$ SDS-polyacrylamide gel electrophoresis gels. Following electrophoresis, the proteins were transferred onto a polyvinylidene fluoride membrane and the membranes were blocked in 5\% skim milk in Tris-HCl buffer salt solution-Tween (TBST) for $2 \mathrm{~h}$ at room temperature. The membranes were then incubated with the following primary antibodies overnight at $4^{\circ} \mathrm{C}$ : Anti-zonula occludens-1 (ZO-1; 61-7300; 1:500; Thermo Fisher Scientific, Inc.), anti-p-NFATc1 (SC32979; 1:500; Santa Cruz Biotechnology, Inc., Dallas, TX, USA), anti-t-NFATc1 (SC13033; 1:500; Santa Cruz Biotechnology, Inc.). $\beta$-actin (3700S; 1:2,000; Cell Signaling Technology, Inc., Danvers, MA, USA) was used as a loading control. The membranes were then incubated with horseradish peroxidase (HRP)-conjugated goat anti-mouse immunoglobulin G secondary antibody (A21010; 1:5,000) and HRP-conjugated goat anti-rabbit IgG secondary antibody (A21020; 1:5,000; both from Abbkine Scientific Co., Ltd., Wuhan, China) for $2 \mathrm{~h}$ at room temperature. The immunoreactive bands were visualized with enhanced chemiluminescence (Pierce; Thermo Fisher Scientific, Inc., Waltham, MA, USA).

Immunofluorescent microscopy. The mRMECs were plated on glass slides in a 12 -well plate at a density of $1 \times 10^{5}$ per well and pretreated with hypoxia and FK506 (10 $\mu \mathrm{M})$. Following washing with PBS, the cells were fixed with $4 \%$ paraformaldehyde for $30 \mathrm{~min}$ at room temperature. The cells were then incubated with polyclonal rabbit primary antibodies against ZO-1 (1:500; Cell Signaling Technology, Inc.) in 5\% bovine serum albumin (BSA; Sigma-Aldrich; Merck KGaA) at $4{ }^{\circ} \mathrm{C}$ overnight, followed by blocking with $5 \%$ BSA for $2 \mathrm{~h}$ at room temperature. The next day, following washing in PBS, the slides were incubated with Alexa Fluor 555 conjugated anti-rabbit IgG secondary antibody (4413S; 1:1,000; Cell Signaling Technology, Inc.) for $2 \mathrm{~h}$ at room temperature, and the nuclei were labeled with 4', 6-diamidino-2-phenylindole for $10 \mathrm{~min}$. Images were captured under a confocal microscope (Zeiss 510; Carl Zeiss AG, Oberkochen, Germany). 
Reverse transcription-quantitative polymerase chain reaction $(R T-q P C R)$ analysis. Total RNA were extracted from the mRMECs using TRIzol reagent (Invitrogen; Thermo Fisher Scientific, Inc.). The purity and concentration of RNA samples were confirmed by the ratio of optical densities at 260 and $280 \mathrm{~nm}$. Then, $1 \mu \mathrm{l}$ RNA was reverse-transcribed in a reaction mixture containing $1 \mathrm{U}$ RNase inhibitor, $500 \mathrm{ng}$ random primers, $3 \mathrm{mM} \mathrm{MgCl} 2,0.5 \mathrm{mM}$ dNTP, $1 \mathrm{X}$ RT buffer and $10 \mathrm{U}$ reverse transcriptase (Promega, Madison, WI, USA). Ths synthesized cDNA was used as a template for PCR reaction using GoTaq polymerase (Promega Corporation, Madison, WI, USA). The RT-qPCR analysis was performed using SYBR-Green qRT-PCR Master mix, according to the manufacturer's protocol (Biotool, Houston, TX, USA). qRT-PCR was performed for 40 cycles of denaturation at $94^{\circ} \mathrm{C}$ for $50 \mathrm{sec}$, annealing at $55^{\circ} \mathrm{C}$ for $50 \mathrm{sec}$ and extension at $72^{\circ} \mathrm{C}$ for $50 \mathrm{sec}$. The quantification cycle values were normalized against $\beta$-actin and the $2^{-\Delta \Delta \mathrm{Cq}}$ method (13) was used to calculate target gene expression. The results determined as the relative value compared with that of the control. The primers for target genes were obtained from the NCBI GeneBank database (http://www. ncbi.nlm.nih.gov/genbank/). The primers for COX-2, inducible nitric oxide synthase (iNOS), monocyte chemotactic protein-1 (MCP-1) and $\beta$-actin were as follows: COX-2 forward, 5'-CCA GAT GAT ATC TTT GGG GAG AC-3' and reverse, 5'-CTT GCA TTG ATG GTG GCT G-3'; iNOS forward, 5'-ACA ACA GGA ACC TAC CAG CTC A-3' and reverse, 5'-GAT GTT GTA GCG CTG TGT GTC A-3'; MCP-1 forward, 5'-ACT GAA GCC AGC TCT CTC TTC CTC-3' and reverse, 5'-TTC CTT CTT GGG GTC AGC ACA GAC-3'; $\beta$-actin forward, 5'-GGC GGA CTA TGA CTT AGT TG-3' and reverse, 5'-AAA CAA CAA TGT GCA ATC AA-3'. The samples were examined in triplicate and the experiment was repeated twice.

Enzyme-linked immunosorbent assay (ELISA). The supernatants of the $\mathrm{mRMEC}$ culture media were harvested $48 \mathrm{~h}$ following FK506 treatment and stored at $-80^{\circ} \mathrm{C}$ until assayed. The levels of IL-6, ICAM-1 and VCAM-1 in the supernatants were measured using an ELISA kit. A 96-well plate containing $100 \mu \mathrm{l}$ of captured antibody per well was incubated overnight at room temperature. Following incubation, the plate was blocked with $1 \%$ BSA in PBS for $1 \mathrm{~h}$ at room temperature. The samples were added $(100 \mu \mathrm{l})$ and incubated for $2 \mathrm{~h}$ at room temperature. Following three washes with $0.05 \%$ Tween-20 in PBS, $100 \mu \mathrm{l}$ of monoclonal antibodies were added to each well for $2 \mathrm{~h}$ at room temperature. Streptavidin-HRP was added to each well and incubated for $20 \mathrm{~min}$ at room temperature. The color reagent $\mathrm{A}\left(\mathrm{H}_{2} \mathrm{O}_{2}\right)$ and color reagent $\mathrm{B}$ (tetramethylbenzidine) substrate solution reactions were terminated at $20 \mathrm{~min}$ with $2 \mathrm{NH}_{2} \mathrm{SO}_{4}$. Each well was immediately read on a microplate reader at $450 \mathrm{~nm}$. The measurements were performed in triplicate.

Statistical analysis. All data are expressed as the mean \pm standard error of the mean of three independent experiments. Differences in mean values among groups were subjected to one-way factorial analysis of variance followed by the Bonferroni post hoc test. $\mathrm{P}<0.05$ was considered to indicate a statistically significant difference. All statistical analyses were performed using SPSS 20.0 software (IBM SPSS, Armonk, NY, USA).

\section{Results}

FK506 treatment attenuates decreased TEER values in hypoxia-induced mRMECs. At 24 and $48 \mathrm{~h}$, the TEER values of the hypoxia groups were markedly lower, compared with the TEER in the control group. Compared with the hypoxia group, the TEER values of the FK506 groups (1 and $10 \mu \mathrm{M})$ were significantly higher, with $10 \mu \mathrm{M}$ exhibiting a more marked effective, compared with $1 \mu \mathrm{M}$ (Fig. 1).

FK506 treatment inhibits increased cell permeability in hypoxia-induced mRMECs. Compared with the control group, the cell permeability of the hypoxia group was increased at 24 and $48 \mathrm{~h}$. However, compared with the hypoxia group, the permeability of cells in the groups treated with FK506 at concentrations of 1 and $10 \mu \mathrm{M}$ were significantly decreased. The reduction in cell permeability in the $10 \mu \mathrm{M}$ group was more marked, compared with that in the $1 \mu \mathrm{M}$ group (Fig. 2).

FK506 treatment attenuates the decreased protein expression of ZO-1 in hypoxia-induced mRMECs. ZO-1 is a protein associated with tight junctions, which is involved in the epithelial function and barrier system. The results of the western blot analysis showed that the expression of ZO-1 was decreased in the mRMECs exposed to hypoxia. However, the protein expression levels of ZO-1 in the 1 and $10 \mu \mathrm{M}$ FK506 groups were increased significantly, with higher expression in the $10 \mu \mathrm{M}$ FK506 group (Fig. 3A and B).

The results of the immunofluorescence analysis were similar. Compared with the control group, the expression of ZO-1 in the hypoxia group was lower. Following treatment with FK506 at a concentration of $10 \mu \mathrm{M}$, the protein expression of ZO-1 was elevated (Fig. 3C). These results showed that FK506 suppressed the damage to tight junctions in hypoxia-induced mRMECs.

FK506 treatment inhibits the increased mRNA expression of COX-2, iNOS, MCP-1 in hypoxia-induced mRMECs. RT-qPCR analysis was performed to examine the mRNA expression levels of COX-2, iNOS and MCP-1 in the control, hypoxia and FK506 groups. As shown in Fig. 4A-C, hypoxia significantly increased the mRNA expression levels of COX-2, iNOS and MCP-1. No significant differences were found between the hypoxia group and the $0.1 \mu \mathrm{M}$ FK506 group. However, compared with the hypoxia group, the mRNA expression levels were significantly decreased in the 1 and $10 \mu \mathrm{M}$ FK506 group, and was more marked in the $10 \mu \mathrm{M}$ group.

FK506 treatment inhibits the increased expression levels of IL-6, ICAM-1 and VCAM-1 in hypoxia-induced mRMECs. ELISA was performed to determine the concentrations of IL-6, ICAM-1 and VCAM-1 in the supernatants. It was found that the concentrations of IL-6, ICAM-1 and VCAM-1 in the hypoxia groups were significantly higher, compared with those in the control groups. The concentrations were decreased following treatment with FK506 (1 and $10 \mu \mathrm{M})$, which was more marked in the $10 \mu \mathrm{M}$ group (Fig. 4D-F).

FK506 treatment attenuates the decreased expression of $p$-NFATcl and the increased expression of $t$-NFATcl 

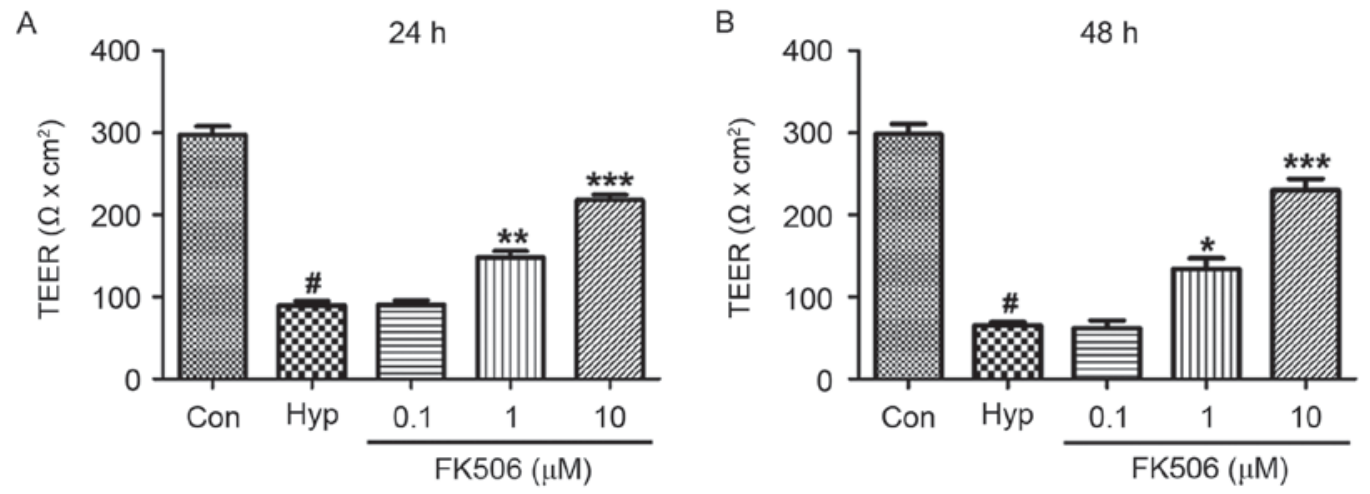

Figure 1. Effect of FK506 on changes of TEER in hypoxia-induced mRMECs. Following culture under hypoxic conditions $\left(93 \% \mathrm{~N}_{2}, 5 \% \mathrm{CO}_{2}, 2 \% \mathrm{O}_{2}\right)$ at $37^{\circ} \mathrm{C}$ for $24 \mathrm{~h}$, the mRMECs were treated with FK506 at concentrations of $0.1,1$ and $10 \mu \mathrm{M}$ for (A) $24 \mathrm{~h}$ and (B) $48 \mathrm{~h}$. The results are representative of three independent experiments and data are expressed as the mean \pm standard error of the mean. ${ }^{*} \mathrm{P}<0.05$, vs. Con; ${ }^{*} \mathrm{P}<0.05,{ }^{* *} \mathrm{P}<0.01$ and ${ }^{* * * *} \mathrm{P}<0.001$, vs. Hyp. TEER, trans-epithelial electrical resistance; mRMECs, mouse retinal microvascular endothelial cells; Con, control; Hyp, hypoxia.

A

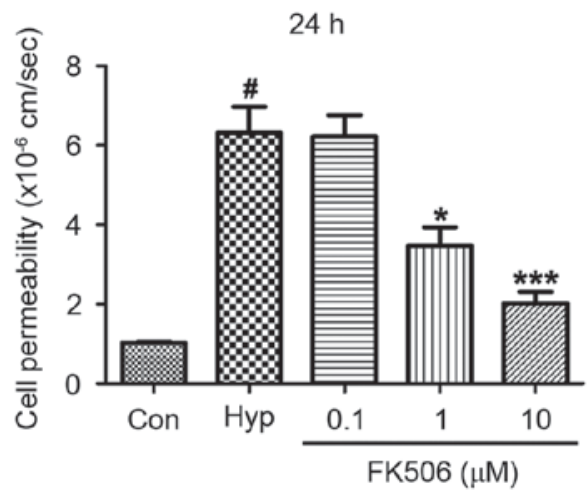

B

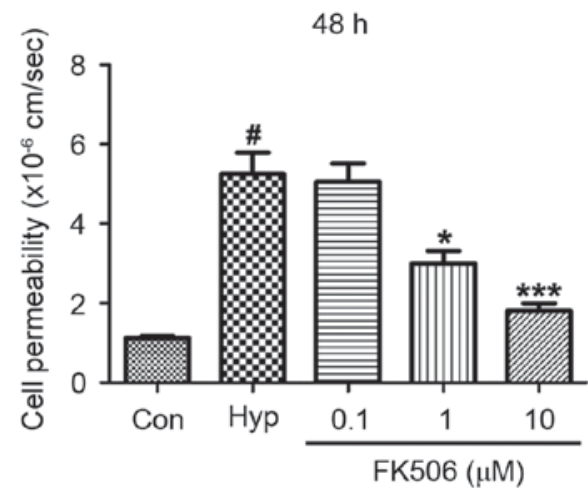

Figure 2. Effect of FK506 on changes of cell permeability in hypoxia-induced mRMECs. Following culture under hypoxia conditions $\left(93 \% \mathrm{~N}_{2}, 5 \% \mathrm{CO}_{2}, 2 \% \mathrm{O}_{2}\right)$ at $37^{\circ} \mathrm{C}$ for $24 \mathrm{~h}$, the mRMECs were treated with FK506 at concentrations of $0.1,1$ and $10 \mu \mathrm{M}$ for (A) $24 \mathrm{~h}$ and (B) $48 \mathrm{~h}$. Cell permeability was then measured with fluorescein isothiocyanate-dextran. The results are representative of three independent experiments and data are expressed as the mean \pm standard error of the mean. ${ }^{\#} \mathrm{P}<0.05$, vs. Con; ${ }^{*} \mathrm{P}<0.05$ and ${ }^{* * *} \mathrm{P}<0.001$, vs. Hyp. mRMECs, mouse retinal microvascular endothelial cells; Con, control; Hyp, hypoxia.

in hypoxia-induced $m R M E C s$. Western blot analysis was performed to examine the protein expression of NFATc1. As shown in Fig. 5, hypoxia significantly increased the protein expression of total (t-)NFATc1, whereas the expression of phosphorylated (p-)NFATc1 was downregulated, compared with the control group. Following treatment with FK506 at concentrations of 1 and $10 \mu \mathrm{M}$, the expression of t-NFATc1 was significantly decreased, whereas the expression of p-NFATc1 was significantly increased, compared with that in the hypoxia group. These changes in protein expression were more marked in the $10 \mu \mathrm{M}$ group.

\section{Discussion}

RMECs, a specialized cell type of retinal vessels, are involved in the physiological and pathological processes of RNV. It has been reported that RMECs are sensitive to hypoxia and that the tight junctions of RMECs are destroyed following exposure to hypoxia. Subsequently, neovascularization appears and several severe symptoms occur, including bleeding, exudation and edema. Angiogenesis is recognized as a crucial factor in RNV diseases, including DR and ROP, which lead to impaired vision. Anti-VEGF is the most effective therapy at present. However, it has restrictions in clinical applications (3).
Therefore, it is important and urgent to identify a more effective and economic therapy.

FK506 is a potent immunosuppressive agent with clinical applications, including transplantation, atopic dermatitis and rheumatoid arthritis (5-7). Investigations on inflammation have shown that FK506 can suppress T cell activation and inflammatory cytokine expression, including IL-2 (8). It can also effectively downregulate the nuclear factor- $\kappa \mathrm{B}$ pathway in various cell types (14-17). Studies on microangiopathy have suggested that FK506 can reduce the high glucose-induced upregulated expression of VEGF in Müller cells (18). In addition, FK506 can inhibit hyperplasia of the vascular wall in the injured arteria carotis communis (19). Evidence has indicated that FK506 can suppress the progress of microangiopathy $(11,12)$, however, the precise effects of FK506 on RMECs remain to be fully elucidated. The results of the present study revealed for the first time, to the best of our knowledge, several important findings concerning the role of FK506 in suppressing hypoxia-induced inflammation and damage to tight junctions in mRMECs.

Previous studies have shown that TEER values decrease and permeability increases following endothelial barrier injury by hypoxia. In the present study, it was found that TEER values were significantly increased and permeability values were significantly decreased following treatment with FK506 (1 and 
A

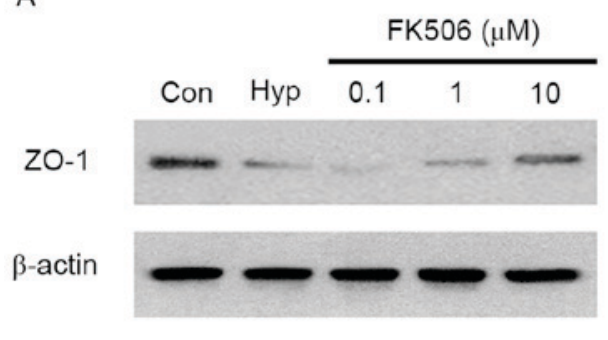

B

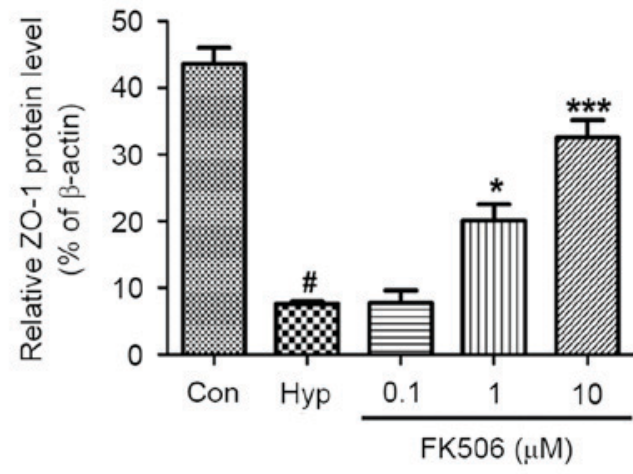

C

$\mathrm{ZO}-1$
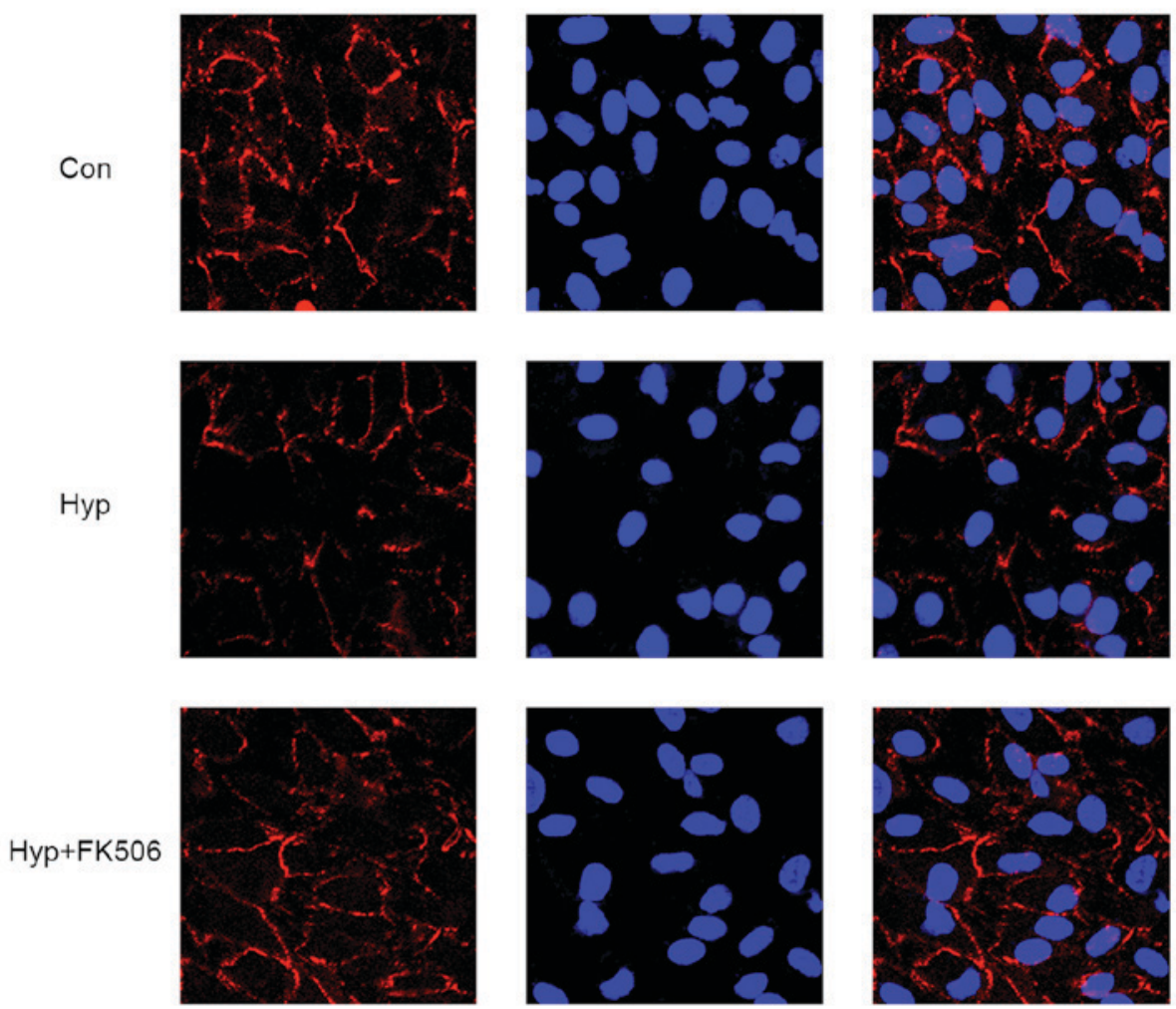

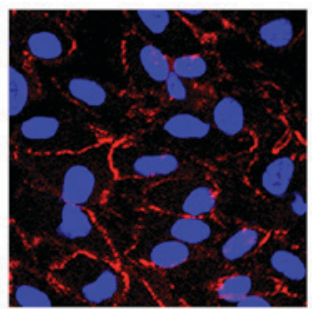

Figure 3. Effect of FK506 on changes of the protein expression of ZO-1 in hypoxia-induced mRMECs. Following culture under hypoxia conditions ( $93 \%$ N $5 \% \mathrm{CO}_{2}, 2 \% \mathrm{O}_{2}$ ) at $37^{\circ} \mathrm{C}$ for $24 \mathrm{~h}$, the mRMECs were treated with FK506 at concentrations of $0.1,1$ and $10 \mu \mathrm{M}$ for $24 \mathrm{~h}$. (A) Protein expression levels of ZO-1 in each group were determined using western blot analysis. $\beta$-actin was used as an internal standard. (B) Quantification of the expression levels of ZO-1 against $\beta$-actin in each group. (C) Protein expression of ZO-1 was also detected using immunofluorescent microscopy (magnification, $\mathrm{x} 40$ ). mRMECs in the FK506 group were treated at $10 \mu \mathrm{M}$, and all groups were stained with ZO-1 (red) and DAPI (blue). The results are representative of three independent experiments and data are expressed as the mean \pm standard error of the mean. ${ }^{*} \mathrm{P}<0.05$, vs. Con; ${ }^{*} \mathrm{P}<0.05$ and ${ }^{* * * *} \mathrm{P}<0.001$, vs. Hyp. mRMECs, mouse retinal microvascular endothelial cells; ZO-1, zonula occludens-1; DAPI, 4', 6-diamidino-2-phenylindole; Con, control; Hyp, hypoxia.

$10 \mu \mathrm{M})$, with the dose of $10 \mu \mathrm{M}$ being more effective. Therefore, it was confirmed that FK506 protected against damage to tight junctions in hypoxia-induced mRMECs. In order to confirm this, western blot analysis and immunofluorescent staining were performed to measure the protein expression levels of ZO-1. ZO-1 is a type of cytoskeletal protein, which is involved in maintaining the stable structure of tight junctions. The present study found that the protein expression of ZO-1 was lower following exposure to hypoxia, however, it was expressed at high levels following treatment with FK506 (1 and $10 \mu \mathrm{M})$. Although 1 and $10 \mu \mathrm{M}$ FK506 were effective, the protein level of ZO-1 was higher in the $10 \mu \mathrm{M}$ group. Consequently, these data suggested that FK506 inhibited hypoxia-induced tight junction damage in the mRMECs.
Previous studies have shown that tight junctions in inflammation are severely damaged. Corneal inflammation has been shown to downregulate tight junction expression and endothelial cell integrity in a mouse model (20). Commonly used anti-inflammatory treatment for patients with inflammatory disease reduces inflammation and maintains normal barrier integrity (21). To determine whether FK506 protects tight junctions in hypoxia-induced $\mathrm{mRMECs}$ by suppressing inflammation, the present study performed RT-qPCR analysis and ELISA to analyze the changes in the expression of of COX-2, iNOS, MCP-1, IL-6, ICAM-1 and VCAM-1, all of which are known to be key factors responsible for hypoxia-induced retinal inflammation and neovascularization. Following exposure to hypoxia, the concentrations of these cytokines 
A

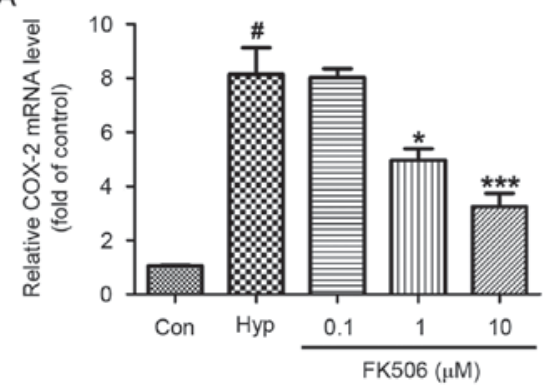

C

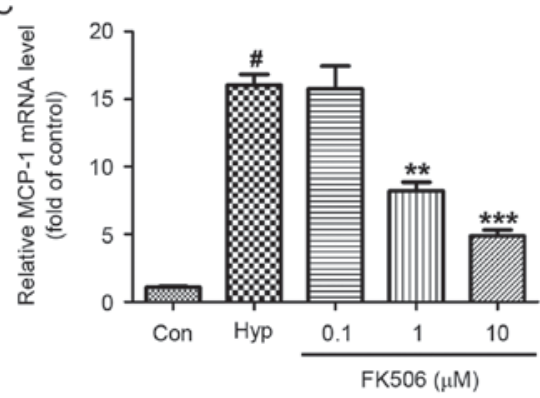

$\mathrm{E}$

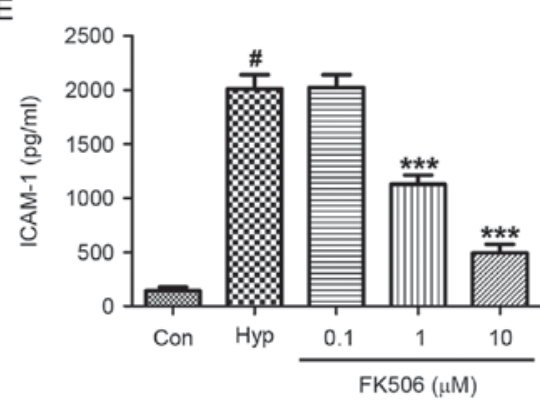

B

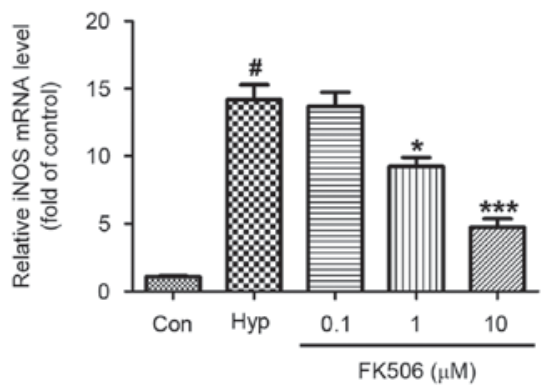

D

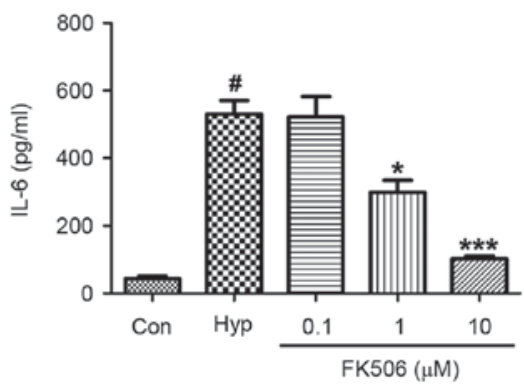

$\mathrm{F}$

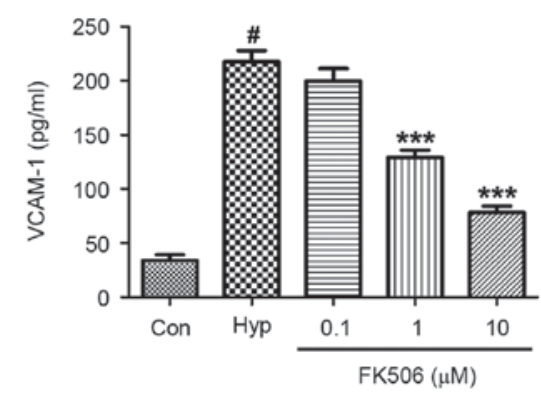

Figure 4. Effect of FK506 on changes of the expression levels of COX-2, iNOS, MCP-1, IL-6, ICAM-1 and VCAM-1 in hypoxia-induced mRMECs. Following culture under hypoxic conditions $\left(93 \% \mathrm{~N}_{2}, 5 \% \mathrm{CO}_{2}, 2 \% \mathrm{O}_{2}\right)$ at $37^{\circ} \mathrm{C}$ for $24 \mathrm{~h}$, the mRMECs were treated with FK506 at concentrations of $0.1,1$ and $10 \mu \mathrm{M}$ for $24 \mathrm{~h}$. The mRNA levels of (A) COX-2, (B) iNOS and (C) MCP-1 were determined using reverse transcription-quantitative polymerase chain reaction analysis against the control group. The expression levels of (D) IL-6, (E) ICAM-1 and (F) VCAM-1 in supernatants were determined using an enzyme-linked immunosorbent assay. The results are representative of three independent experiments and data are expressed as the mean \pm standard error of the mean. ${ }^{~} \mathrm{P}<0.05$, vs. Con; ${ }^{*} \mathrm{P}<0.05,{ }^{* *} \mathrm{P}<0.01$ and ${ }^{* * * *} \mathrm{P}<0.001$, vs. Hyp. mRMECs, mouse retinal microvascular endothelial cells; COX-2, cyclooxygenase-2; iNOS, inducible nitric oxide synthase; MCP-1, monocyte chemotactic protein-1; IL-6, interleukin-6; ICAM-1, intercellular cell adhesion molecule-1; VCAM-1, vascular cell adhesion molecule-1; Con, control; Hyp, hypoxia.
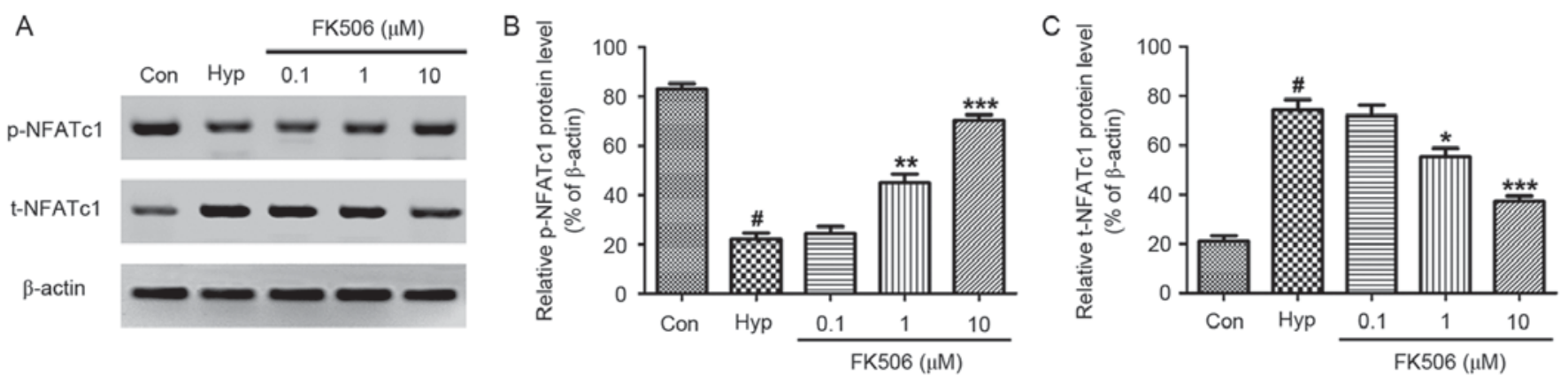

Figure 5. Effect of FK506 on changes of the expression of NFATc1 in hypoxia-induced mRMECs. Following culture under hypoxic conditions $\left(93 \% \mathrm{~N}_{2}, 5 \% \mathrm{CO}_{2}\right.$, $2 \% \mathrm{O}_{2}$ ) at $37^{\circ} \mathrm{C}$ for $24 \mathrm{~h}$, the mRMECs were treated with FK506 at concentrations of $0.1,1$ and $10 \mu \mathrm{M}$ for $24 \mathrm{~h}$. (A) Protein expression levels of p-NFATc1 and t-NFATc1 in each group were determined using western blot analysis. $\beta$-actin was used as internal standard. (B) Quantification of the expression levels of p-NFATc1 against $\beta$-actin in each group. (C) Quantification of the expression levels of t-NFATc1 against $\beta$-actin in each group. The results are representative of three independent experiments and data are expressed as the mean \pm standard error of the mean. ${ }^{*} \mathrm{P}<0.05$, vs. Con; ${ }^{*} \mathrm{P}<0.05,{ }^{* *} \mathrm{P}<0.01$ and ${ }^{* * * *} \mathrm{P}<0.001, \mathrm{vs}$. Hyp. mRMECs, mouse retinal microvascular endothelial cells; NFATc1, nuclear factor of activated T-cells 1; p-NFATc1, phosphorylated-NFATc1; t-NFATc1, total NFATc1; Con, control; Hyp, hypoxia.

increased markedly. However, treatment with FK506 (1 and $10 \mu \mathrm{M})$ significantly reduced the levels of all these cytokines.
These findings indicated that FK506 protected tight junctions by suppressing hypoxia-induced inflammation in mRMECs. 
NFATc1 proteins are calcineurin-dependent, rapidly inducible transcription factors. Calcium mobilization and the activation of calcineurin leads to NFATc1 dephosphorylation, followed by its translocation into the nucleus where it acts as a transcription factor (22). NFATc1 proteins are present predominantly in lymphoid cells and are involved in different cellular functions, including the immune response, cell proliferation, and development and differentiation, in addition to pathological events, including cancer $(23,24)$. In previous years, several studies have shown that the CaN-NFATc1 signaling pathway not only regulates the growth of the cardiac system (25) and peripheral vasculature (26), but is also involved in the pathological neovascularization of proliferative vitreoretinopathy (27) and tumors (28). In 2013, Bretz et al demonstrated that VEGF induces NFATc1 to transfer into the nucleus in RMECs. Inhibitors of NFATc1 and CaN inhibited RMEC proliferation and vessel formation and, in a model of oxygen-induced retinopathy, the area of retinal neovasculature reduced following intravitreal injection of CaN-NFATc1 interaction inhibitor-6 and FK506 (27). Therefore, the CaN-NFATc1 signaling pathway is crucial in RNV. Based on these findings, the present study hypothesized that FK506 suppresses hypoxia-induced inflammation and protects tight junction function in mRMECs via the CaN-NFATc1 signaling pathway. The results of the present study revealed that the levels of t-NFATc1 were increased in hypoxia, whereas the levels of p-NFATc1 were decreased. Following treatment with FK506, the levels of t-NFATc1 were decreased and levels of p-NFATc1 were increased.

In conclusion, the results of the present study showed that FK506 suppressed hypoxia-induced inflammation and protected tight junction function via the CaN-NFATc1 signaling pathway in mRMECs. FK506 may be a potential effective therapy for hypoxia-induced microangiopathy.

\section{Acknowledgements}

This study was financially supported by the Chinese government in the form of the National Natural Science Foundation of China (grant no. 81670873).

\section{References}

1. Gliem M, Finger RP, Fimmers R, Brinkmann CK, Holz FG and Charbel Issa P: Treatment of choroidal neovascularization due to angioid streaks: A comprehensive review. Retina 33: 1300-1314, 2013.

2. van Lookeren Campagne M, LeCouter J, Yaspan BL and Ye W: Mechanisms of age-related macular degeneration and therapeutic opportunities. J Pathol 232: 151-164, 2014.

3. Mintz-Hittner HA, Kennedy KA and Chuang AZ; BEAT-ROP Cooperative Group: Efficacy of intravitreal bevacizumab for stage 3+ retinopathy of prematurity. N Engl J Med 364: 603-615, 2011.

4. Kollias AN and Ulbig MW: Diabetic retinopathy: Early diagnosis and effective treatment. Dtsch Arztebl Int 107: 75-83, 2010

5. Todo S, Fung JJ, Starzl TE, Tzakis A, Demetris AJ, Kormos R, Jain A, Alessiani M, Takaya S and Shapiro R: Liver, kidney and thoracic organ transplantation under FK 506. Ann Surg 212 295-307, 1990

6. Baumgart DC, Pintoffl JP, Sturm A, Wiedenmann B and Dignass AU: Tacrolimus is safe and effective in patients with severe steroid-refractory or steroid-dependent inflammatory bowel disease-a long-term follow-up. Am J Gastroenterol 101: 1048-1056, 2006.

7. Thestrup-Pedersen K: Tacrolimus treatment of atopic eczema/ dermatitis syndrome. Curr Opin Allergy Clin Immunol 3: 359-362, 2003 .
8. Weiwad M, Edlich F, Kilka S, Erdmann F, Jarczowski F, Dorn M, Moutty MC and Fischer G: Comparative analysis of calcineurin inhibition by complexes of immunosuppressive drugs with human FK506 binding proteins. Biochemistry 45: 15776-15784, 2006.

9. Haydon GH and Hayes PC: New immunosuppressive treatment in transplantation medicine. Baillieres Clin Gastroenterol 8: 455-464, 1994.

10. Tsuzuki S, Toyama-Sorimachi N, Kitamura F, Tobita Y and Miyasaka M: FK506 (tacrolimus) inhibits extravasation of lymphoid cells by abrogating VLA-4/VCAM-1 mediated transendothelial migration. FEBS Lett 430: 414-418, 1998.

11. Kahan BD: Cyclosporine. N Engl J Med 321: 1725-1738, 1989.

12. Jacobson P, Uberti J, Davis W and Ratanatharathorn V: Tacrolimus: A new agent for the prevention of graft-versus-host disease in hematopoietic stem cell transplantation. Bone Marrow Transplant 22: 217-225, 1998.

13. Livak KJ and Schmittgen TD: Analysis of relative gene expression data using real-time quantitative PCR and the 2(-Delta Delta C(T)) method. Methods 25: 402-408, 2001.

14. Zeyda M, Geyeregger R, Poglitsch M, Weichhart T, Zlabinger GJ, Koyasu S,Hörl WH, Stulnig TM, Watschinger B and Saemann MD: Impairment of $\mathrm{T}$ cell interactions with antigen-presenting cells by immunosuppressive drugs reveals involvement of calcineurin and NF-kappaB in immunological synapse formation. J Leukoc Biol 81: 319-327, 2007.

15. Du S, Hiramatsu N, Hayakawa K, Kasai A, Okamura M, Huang T, Yao J, Takeda M, Araki I, Sawada N, et al: Suppression of NF-kappaB by cyclosporin a and tacrolimus (FK506) via induction of the C/EBP family: Implication for unfolded protein response. J Immunol 182: 7201-7211, 2009.

16. Yoshino T, Nakase H, Honzawa Y, Matsumura K, Yamamoto S, Takeda Y, Ueno S, Uza N, Masuda S, Inui K and Chiba T: Immunosuppressive effects of tacrolimus on macrophages ameliorate experimental colitis. Inflamm Bowel Dis 16: 2022-2033, 2010.

17. Nakamura-Yanagidaira T, Takahashi Y, Sano K, Murata T and Hayashi T: Development of spontaneous neuropathy in $\mathrm{NF}-\kappa \mathrm{Bp} 50$-deficient mice by calcineurin-signal involving

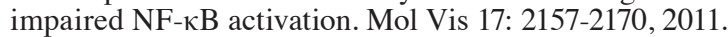

18. Xia W, Xia J, Zhang XF, Zhong L, Sun ZT and Wang YM: Inhibition of FK506 on the expression of vascular endothelial growth factor in retinal Müller cells cultured by high concentration glucose. Chin J Exp Ophthalmol 32: 998-1003, 2014.

19. Xiao ZX, Xie YY and Zhen RD: Effect of FK506 on injured vascular wall. Hainan Med J 4: 1-3, 2009 (In Chinese).

20. Larkin DF: Longitudinal changes to tight junction expression and endothelial cell integrity in a mouse model of sterile corneal inflammation. Invest Ophthalmol Vis Sci 57: 3485, 2016.

21. Cannon AR, Akhtar S, Hammer AM, Morris NL, Javorski MJ, Li X, Kennedy RH, Gamelli RL and Choudhry MA: Effects of mesalamine treatment on gut barrier integrity after burn injury. J Burn Care Res 37: 283-292, 2016.

22. Rao A, Luo C and Hogan PG: Transcription factors of the NFAT family: Regulation and function. Annu Rev Immunol 15: 707-747, 1997.

23. Hogan PG, Chen L, Nardone J and Rao A: Transcriptional regulation by calcium, calcineurin, and NFAT. Genes Dev 17: 2205-2232, 2003

24. Mancini M and Toker A: NFAT proteins: Emerging roles in cancer progression. Nat Rev Cancer 9: 810-820, 2009.

25. Chang CP, Neilson JR, Bayle JH, Gestwicki JE, Kuo A, Stankunas K, Graef IA and Crabtree GR: A field of myocardial-endocardial NFAT signaling underlies heart valve morphogenesis. Cell 118: 649-663, 2004.

26. Graef IA, Chen F, Chen L, Kuo A and Crabtree GR: Signals transduced by $\mathrm{Ca}(2+) /$ calcineurin and NFATc $3 / \mathrm{c} 4$ pattern the developing vasculature. Cell 105: 863-875, 2001.

27. Bretz CA, Savage S, Capozzi M and Penn JS: The role of the NFAT signaling pathway in retinal neovascularization. Invest Ophthalmol Vis Sci 54: 7020-7027, 2013.

28. Minami T, Jiang S, Schadler K, Suehiro J, Osawa T, Oike Y, Miura M, Naito M, Kodama T and Ryeom S: The calcineurin-NFAT-angiopoietin-2 signaling axis in lung endothelium is critical for the establishment of lung metastases. Cell Rep 4: 709-723, 2013 